\title{
Hexose-6-phosphate dehydrogenase: a new risk gene for multiple sclerosis
}

\author{
Antonio Alcina ${ }^{1}$, Sreeram V Ramagopalan ${ }^{2}$, Óscar Fernández ${ }^{3}$, Antonio Catalá-Rabasa ${ }^{1}$, María Fedetz ${ }^{1}$, \\ Dorothy Ndagire $^{1}$, Laura Leyva ${ }^{3}$, Carmen Arnal ${ }^{4}$, Concepción Delgado $^{5}$, Miguel Lucas ${ }^{6}$, Guillermo Izquierdo $^{7}$, \\ George C Ebers ${ }^{2}$ and Fuencisla Matesanz ${ }^{\star, 1}$
}

\begin{abstract}
A recent genome-wide association study (GWAS) performed by the The Wellcome Trust Case-Control Consortium based on 12374 nonsynonymous single-nucleotide polymorphisms (SNPs) provided evidence for several genes involved in multiple sclerosis (MS) susceptibility. In this study, we aimed at verifying the association of 19 SNPs with MS, with $P$-values $\leq 0.005$, in an independent cohort of 732 patients and 974 controls, all Caucasian from the South of Spain. We observed an association of the rs 17368528 polymorphism with MS $(P=0.04$, odds ratio $(O R)=0.801,95 \%$ confidence interval $(C l)=0.648-0.990)$. The association of this polymorphism with MS was further validated in an independent set of 1318 patients from the Canadian Collaborative Project $(P=0.04,0 R=0.838,95 \% \mathrm{Cl}=0.716-0.964)$. This marker is located on chromosome $1 \mathrm{p} 36.22$, which is $1 \mathrm{Mb}$ away from the MS-associated kinesin motor protein KIF1B, although linkage disequilibrium was not observed between these two markers. The rs17368528 SNP results in an amino-acid substitution (proline to leucine) in the fifth exon of the hexose-6-phosphate dehydrogenase (H6PD) gene, in which some variants have been reported to attenuate or abolish H6PD activity, in individuals with cortisone reductase deficiency. This study corroborates the association of one locus determined by GWAS and points to H6PD as a new candidate gene for MS.
\end{abstract}

European Journal of Human Genetics (2010) 18, 618-620; doi:10.1038/ejhg.2009.213; published online 25 November 2009

Keywords: multiple sclerosis (MS); polymorphisms; genetics; H6PD gene; 1p36.22; association

\section{INTRODUCTION}

Multiple sclerosis (MS) is a chronic autoimmune disease with a complex pathogenesis, in which demyelination and neurodegeneration are the main contributors to disability. ${ }^{1}$ Susceptibility to MS is thought to be conferred by a combination of genetic and environmental factors. ${ }^{2}$ The most established region in predisposition to MS is the major histocompatibility complex on chromosome 6p21, specifically, the HLA-DRB1*1501 class II allele. ${ }^{3}$ Currently, other loci have been identified that have convincing evidence for an association with MS, such as the IL2RA, ${ }^{4,5}$ IL7RA, ${ }^{6,7} \mathrm{KIF} 1 B,{ }^{8} \mathrm{IRF} 5,{ }^{9} \mathrm{EVI} 5,{ }^{4,10}$ $C D 226^{11,12}$ and CLEC16A ${ }^{12}$ genes. Some of these genes were identified through a classical candidate gene approach; however, many new candidate risk factors arise from the hypothesis-free approach provided by genome-wide association studies (GWAS). The Wellcome Trust Case-Control Consortium (WTCCC) carried out a GWAS based on 12374 nonsynonymous single-nucleotide polymorphisms (SNPs) typed in 975 patients and 1466 controls. ${ }^{13}$ No SNP outside the HLA region provided a significant association with MS at the genome-wide level in this study. However, the initial screen yielded promising signals. One of the signals located at the tyrosine kinase 2 (TYK2) gene has since been confirmed with an independent cohort. ${ }^{14}$

In this study, we assessed the risk contribution of 19 SNPs that showed some degree of association in the GWAS performed by the WTCCC using MS patients and controls from the south of Spain. We identified hexose-6-phosphate dehydrogenase ( $H 6 P D$, glucose-1-dehydrogenase) as a novel risk gene for MS and validated this finding in an independent cohort of Canadian MS patients.

\section{MATERIALS AND METHODS}

Subjects

Case samples comprised 732 patients with clinically definite MS, according to Poser's criteria. ${ }^{15}$ They were obtained from four public hospitals: the Hospital Clínico in Granada $(n=126)$, the Hospital Virgen de las Nieves in Granada $(n=165)$, the Hospital Carlos Haya in Málaga $(n=365)$ and the Hospital Virgen de la Macarena in Seville $(n=76)$. All three cities are within a $200 \mathrm{~km}$ radius in the South of Spain. The mean age $( \pm S D)$ of cases at the time of sample collection was $29.84 \pm 10.66$ years and that of controls was $33.43 \pm 12.19$ years. The percentage of females was $68 \%$ for cases and $68 \%$ for controls. All patients were classified as RR (relapsing remitting) or SP (secondary progressive) MS cases. Controls were 974 blood donors with no history of inflammatory disease attending the blood banks of Granada $(n=823)$, Seville $(n=71)$ and Málaga $(n=80)$. Both cases and controls were Caucasians. The study was approved by the Ethics Committees of each of the hospitals participating in the study and a written informed consent was obtained from all participants.

Genotyping of individuals from the Spanish population In this analysis, we included the 19 SNPs identified in the WTCCC GWAS with a $P$-value $\leq 0.005$ from all chromosomes, except chromosome 6 . We typed all 19 SNPs in 732 patients and 974 controls. Genotyping was performed using

\footnotetext{
1Department of Cell Biology and Immunology, Instituto de Parasitología y Biomedicina 'López Neyra', Consejo Superior de Investigaciones Científicas, Granada, Spain; 2Department of Clinical Neurology and The Wellcome Trust Centre for Human Genetics, University of Oxford, Oxford, UK; ${ }^{3}$ Servicio de Neurología, Instituto de Neurociencias Clínicas, Hospital Carlos Haya, Málaga, Spain; ${ }^{4}$ Servicio de Neurología, Hospital Virgen de las Nieves, Granada, Spain; ${ }^{5}$ Centro Regional de Transfusión Sanguínea GranadaAlmería, Granada, Spain; ${ }^{6}$ Servicio de Biología Molecular, Hospital Virgen Macarena, Sevilla, Spain; ${ }^{7}$ Unidad de Esclerosis Múltiple, Hospital Virgen Macarena, Sevilla, Spain *Correspondence: Dr F Matesanz, Department of Cell Biology and Immunology, Instituto de Parasitología y Biomedicina Lopez Neyra, CSIC, Parque Tecnológico de Ciencias de la Salud, Avda, del Conocimiento s/n, 18100, Granada, Spain. Tel: + 34958 181668; Fax: + 34958 181632; E-mail: pulgoso@ipb.csic.es

Received 23 June 2009; revised 10 September 2009; accepted 20 October 2009; published online 25 November 2009
} 
the MassARRAY SNP genotyping system (Sequenom, San Diego, CA, USA) according to the manufacturer's instructions.

This genotyping platform is at the Spanish National Genotyping Center's facilities in Santiago de Compostela University, Galicia, Spain. As quality control, they genotyped a trio of CEPH samples (CEPH pedigree 1340; samples NA07019, NA07029 and y NA07062). The genotypes of these samples resulted in no detection of Mendelian inconsistencies and corresponded with the ones deposited in HapMap. On the other hand, $4 \%$ of random samples were subjected to regenotyping. The resulting data were concordant with an average accuracy of $>99.9 \%$.

\section{Genotyping of participants from The Canadian Collaborative Project on the Genetic Susceptibility to MS}

A total of 1318 individuals with definite MS and 1507 of their unaffected first-degree relatives were typed for rs17368528, rs10489990 and rs3746887 as part of the Canadian Collaborative Project on the Genetic Susceptibility to MS (CCPGSMS). ${ }^{16}$ Genotyping was carried out using the MassARRAY system/Homogeneous MassExtend assay, following the protocol provided by Sequenom. All genotypes were generated blind to the pedigree structure and disease status of the individual.

\section{Statistical analysis of data from the Spanish population}

A $\chi^{2}$-test $(P>0.05)$ was used to assess whether genotypic distributions fulfilled the Hardy-Weinberg equilibrium. PLINK software (http://pngu.mgh.harvard. edu/ purcell/plink/) was used to compare the allele counts in cases and controls by Fisher's exact test and to calculate odds ratios (ORs) with $95 \%$ confidence intervals (CIs). Power was calculated for the size of effect detected in the WTCCC GWAS at an $\alpha$-level of 0.05 , assuming a multiplicative allelic effects model, using 732 cases and 974 controls.

\section{Statistical analysis of data from the Canadian Collaborative Project} on the Genetic Susceptibility to MS

Transmission disequilibrium testing was performed using the PLINK analysis package. For transmission disequilibrium tests, the $\chi^{2}$ distribution was used to assess significance. The effects of rs10492972 and rs17368525 on the risk of MS were assessed by regression using the $\mathrm{R}$ statistical package (www.r-project.org).

\section{RESULTS}

A total of 19 MS-associated variants, with a $P$-value $\leq 0.005$, reported by WTCCC GWAS were selected. The polymorphisms were first genotyped in a Spanish cohort of MS patients $(n=732)$ and controls $(n=974)$. No marker showed deviation from the Hardy-Weinberg equilibrium. Three of the 19 polymorphisms showed significant signals of association with MS $(P<0.05)$ (Table 1$)$. However, the association for rs10489990 and rs3746887 was with the opposite allele in the Spanish cohort as compared with the original study. To replicate this finding, we genotyped these three SNPs in an independent family cohort containing more than 1300 patients from the Canadian Collaborative Project on the Genetic Susceptibility to MS. Only the association for SNP, rs17368528, was replicated in this study $(P=0.04$, $\mathrm{OR}=0.83,95 \% \mathrm{CI}=0.72-0.96$ ), as indicated in Table 2. The SNP rs 17368528 is a nonsynonymous (leucine/proline) SNP localized in the fifth exon of H6PD (glucose 1-dehydrogenase) gene. This marker is located on chromosome $1 \mathrm{p} 36.22$, which is $1 \mathrm{Mb}$ away from the MS-associated KIF1B gene. The linkage disequilibrium (LD) value between these two markers was $r^{2}=0$ for the Spanish cohort and $r^{2}=0.21$ for the Canadian cohort, and the association of H6PD was independent of the KIF1B-associated SNP (rs10492972), as assessed by logistic regression analysis $(P<0.05$ for both SNPs).

\section{DISCUSSION}

In MS, the current number of markers in GWAS with evidence for association exceeding a genome-wide significance threshold is small.

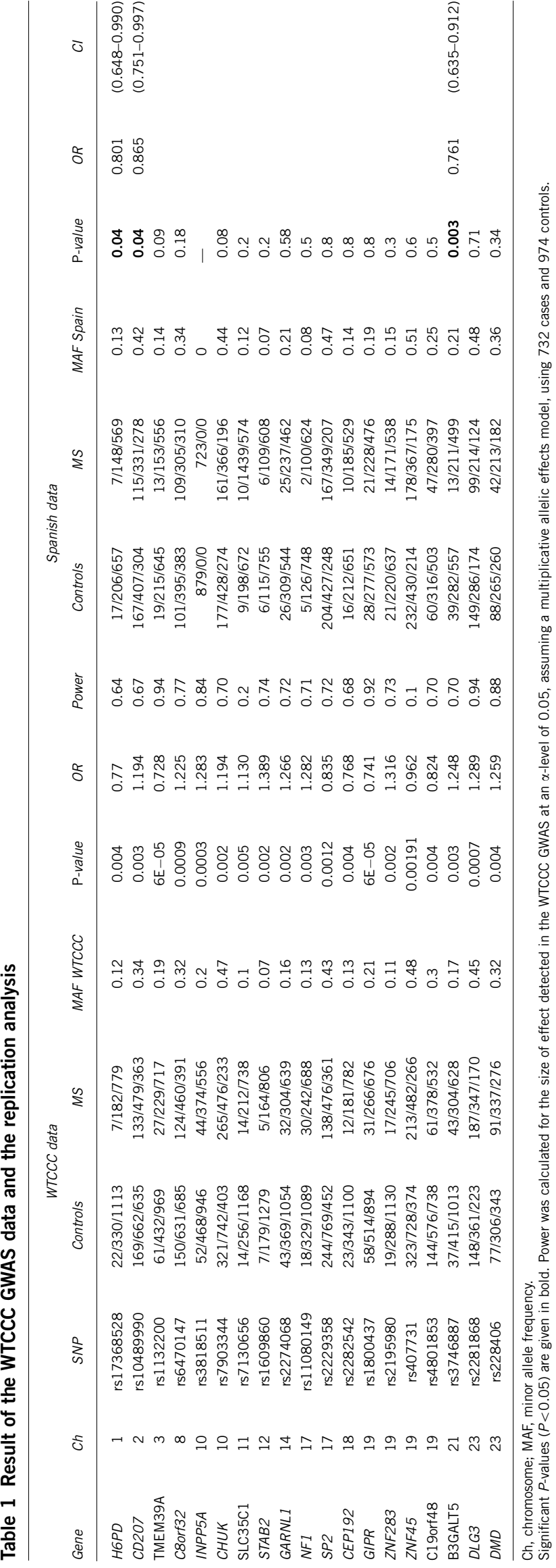


Table 2 Results of replication of the three SNPs associated with the Spanish population in the Canadian cohort

\begin{tabular}{lcccll}
\hline SNP & MA & TR:NT & \multicolumn{1}{c}{ OR $(95 \% \mathrm{Cl})$} & $\chi^{2}$-test & P-value \\
\hline rs17368528 & T & $239: 285$ & $0.838(0.716-0.964)$ & 4.038 & 0.0445 \\
rs10489990 & A & $566: 537$ & $1.054(0.897-1.232)$ & 0.7623 & 0.3826 \\
rs3746887 & T & $324: 327$ & $0.991(0.847-1.161)$ & 0.01382 & 0.9064 \\
\hline
\end{tabular}

MA, minor allele; TR:NT, transmitted: not transmitted.

Markers that do not exceed this threshold are generally neglected, as they may represent false positives. However, some of the markers with a level of significance $\leq P=0.05$ may reflect true associations, given the current modest sample size used in initial MS GWA screens. In this study, we aimed to verify the association of 19 SNPs with MS, with $P$-values of association $<0.005$ reported in the WTCCC GWAS, ${ }^{13}$ and found evidence of association for the rs17368528 SNP located on chromosome 1p36.22 in the Spanish and Canadian Cohorts.

The KIF1B gene, which is also located in the 1 p36.22 locus, has been previously associated with MS susceptibility. ${ }^{8}$ The KIF1B gene is an excellent candidate for MS, as it is required for the localization of MBP (myelin basic protein) mRNA to processes myelinating oligodendrocytes in zebrafish. ${ }^{17}$ However, our data indicate that both markers are independently associated by regression analysis.

$H 6 P D$ is a good candidate for MS, as it encodes an enzyme that catalyzes the initial steps of the pentose phosphate pathway (PPP) within the lumen of the endoplasmic reticulum, providing reducing equivalents such as $\mathrm{NADPH}\left(\mathrm{H}^{+}\right)$. In neurons, most PPP-derived $\operatorname{NADPH}\left(\mathrm{H}^{+}\right)$is used for the regenerating pathway of the antioxidant glutathione $(\mathrm{GSH}) .{ }^{18}$ The decrease in H6PD activity could alter the apoptotic processes in neurons affecting cytochrome $\mathrm{C}$ reduction by GSH. This could perhaps lead to neurodegeneration. ${ }^{19}$ In fact, the missense polymorphism (Arg-Gln) rs6688832, also in the fifth exon of the H6PD gene and in LD $\left(D^{\prime}=0.49\right)$ with the SNP reported here, produces a reduction of $50 \%$ in enzyme activity in individuals with cortisone reductase deficiency. ${ }^{20}$

In summary, we report here an association with the H6PD gene, first identified by a GWAS and confirmed in two large cohorts of MS patients from Spain and Canada. The H6PD gene is an interesting candidate for MS for its possible role in neurodegeneration.

\section{CONFLICT OF INTEREST}

The authors declare no conflict of interest.

\section{ACKNOWLEDGEMENTS}

We thank the patients with multiple sclerosis and persons who acted as controls for making this study possible. Financial support for the study was provided by the Ministerio de Ciencia e Innovación and Fondos Europeos de Desarrollo Regional (FEDER) (grants PN-SAF2006-02023 and PN-SAF2009-11491) and
Junta de Andalucía (P07-CVI-02551) to A Alcina, and Fondo de Investigación Sanitaria (FIS, grant number PI081636) and Servicio Andaluz de Salud (PI0168-2007) to F Matesanz. The Multiple Sclerosis Society of the United Kingdom and the Multiple Sclerosis Society of Canada Scientific Research Foundation support the Canadian Collaborative Study Group. SNP genotyping services were provided by the Spanish 'Centro Nacional de Genotipado CEGEN-USC, www.cegen.org. María Fedetz is a holder of a fellowship from Fundación IMABIS. Dorothy Ndagire is a holder of Agencia Española Cooperación Internacional (AECI)-Ministerio de Asuntos Exteriores fellowship.

1 Hauser SL, Oksenberg JR: The neurobiology of multiple sclerosis: genes, inflammation, and neurodegeneration. Neuron 2006; 52: 61-76.

2 McElroy JP, Oksenberg JR: Multiple sclerosis genetics. Curr Top Microbiol Immunol 2008; 318: 45-72.

3 Ramagopalan SV, Knight JC, Ebers GC: Multiple sclerosis and the major histocompatibility complex. Curr Opin Neurol 2009; 22: 219-225.

4 Matesanz F, Caro-Maldonado A, Fedetz M et al: IL2RA/CD25 polymorphisms contribute to multiple sclerosis susceptibility. J Neurol 2007; 254: 682-684.

5 International Multiple Sclerosis Genetics Consortium: Risk alleles for multiple sclerosis identified by a genomewide study. N Eng/ J Med 2007; 357: 851-862.

6 Gregory SG, Schmidt S, Seth P et al: Interleukin 7 receptor alpha chain (IL7R) shows allelic and functional association with multiple sclerosis. Nat Genet 2007; 39: 1083-1091.

7 Lundmark F, Duvefelt K, lacobaeus E et al: Variation in interleukin 7 receptor alpha chain (IL7R) influences risk of multiple sclerosis. Nat Genet 2007; 39: 1108-1113.

8 Aulchenko YS, Hoppenbrouwers IA, Ramagopalan SV et al: Genetic variation in the KIF1B locus influences susceptibility to multiple sclerosis. Nat Genet 2008; 40: 1402-1403.

9 Kristjansdottir G, Sandling JK, Bonetti A et al: Interferon regulatory factor 5 (IRF5) gene variants are associated with multiple sclerosis in three distinct populations. J Med Genet 2008; 45: 362-369.

10 Hoppenbrouwers IA, Aulchenko YS, Ebers GC et al: EVI5 is a risk gene for multiple sclerosis. Genes Immun 2008; 9: 334-337.

11 Hafler JP, Maier LM, Cooper JD et al: CD226 Gly307Ser association with multiple autoimmune diseases. Genes Immun 2009; 10: 5-10.

12 International Multiple Sclerosis Genetics Consortium (IMSGC): The expanding genetic overlap between multiple sclerosis and type I diabetes. Genes Immun 2009; 10: 11-14.

13 Wellcome Trust Case Control Consortium: Association scan of 14500 nonsynonymous SNPs in four diseases identifies autoimmunity variants. Nat Genet 2007; 39: 1329-1337.

14 Ban M, Goris A, Lorentzen AR et al: Replication analysis identifies TYK2 as a multiple sclerosis susceptibility factor. Eur J Hum Genet 2009; 17: 1309-1313.

15 Poser CM, Paty DW, Scheinberg L et al: New diagnostic criteria for multiple sclerosis: guidelines for research protocols. Ann Neurol 1983; 13: 227-231.

16 Sadovnick AD, Risch NJ, Ebers GC: Canadian collaborative project on genetic susceptibility to MS, phase 2: rationale and method. Canadian Collaborative Study Group. Can J Neurol Sci 1998; 25: 216-221.

17 Lyons DA, Naylor SG, Scholze A, Talbot WS: Kif1b is essential for mRNA localization in oligodendrocytes and development of myelinated axons. Nat Genet 2009; 41: 854-858.

18 Kletzien RF, Harris PKV, Foellmi LA: Glucose-6-phosphate dehydrogenase: a housekeeping enzyme subject to tissue-specific regulation by hormones, nutrients, and oxidant stress. FASEB J 1994; 8: 174-181.

19 Vaughn AE, Deshmukh M: Glucose metabolism inhibits apoptosis in neurons and cancer cells by redox inactivation of cytochrome C. Nat Cell Biol 2008; 10: 1477-1483.

20 Draper N, Walker EA, Bujalska IJ et al: Mutations in the genes encoding 11 betahydroxysteroid dehydrogenase type 1 and hexose-6-phosphate dehydrogenase interact to cause cortisone reductase deficiency. Nat Genet 2003; 34: 434-439. 